\title{
The Prevalence of Sarcopenia Using Different Formulas in Patients with Prediabetes
}

\author{
Sabah Tüzün' (D), Elif Sevinç² (D), İsmet Tamer' (D), Ekrem Orbay' (D), Reșat Dabak' (1) \\ 'Department of Family Medicine, Dr. Lütfi Kırdar Kartal Training and Research Hospital, İstanbul, Turkey \\ ${ }^{2}$ Clinic of Family Medicine, Tokat Reșadiye Government Hospital, Tokat, Turkey
}

ORCID IDs of the authors: S.T. 0000-0002-8859-934X; E.S. 0000-0002-6459-363X; i.T. 0000-000I-9596-7203; E.0. 0000-000I-5933-6965; R.D. 0000-0002-0200-5409.

Cite this article as: Tüzün S, Sevinç E, Tamer I, Orbay E, Dabak R. The Prevalence of Sarcopenia Using Different Formulas in Patients with Prediabetes. Cyprus J Med Sci 2019; 4(I): I-4.

\section{BACKGROUND/AIMS}

Prediabetes is one of the causes of sarcopenia. In addition to the absence of a definite formula to make the diagnosis of sarcopenia, there are also limitations in assessing the muscle mass in obese subjects. The aim of the present study was to evaluate the prevalence of sarcopenia using different formulas in patients with prediabetes.

\section{MATERIAL and METHODS}

Participants with prediabetes who visited the obesity outpatient clinic between 2013 and 2015 were retrospectively evaluated. Muscle mass ratio (MMR) was calculated as the percentage of total muscle mass divided by body weight, whereas skeletal muscle index (SMI) was calculated as the total muscle mass divided by the square of the height. In addition, the percentage of appendicular lean mass (ALM) divided by body weight, the ratio of ALM divided by the square of the height, and the ratio of ALM divided by body mass index (BMI) were also evaluated.

\section{RESULTS}

A total of 524 patients with prediabetes were enrolled into the study. The prevalence of sarcopenia in female patients was 60 (I8.I3\%) using MMR, whereas no sarcopenia was detected using ALM/BMl ratio, SMl, and ALM/height². Sarcopenia was detected in 63 (32.64\%) male patients using MMR, in $17(8.81 \%)$ using ALM/BMl ratio, and in I (0.52\%) using each of SMI and ALM/height².

\section{CONCLUSION}

The prevalence of sarcopenia varies even using ALM/BMI ratio and MMR, which are recommended for evaluation of muscle mass particularly in obese patients with prediabetes. New formulas need to be developed for evaluation of muscle mass to prevent the concealment of sarcopenia in middle-aged patients with prediabetes.

Keywords: Sarcopenia, muscle tissue, prediabetes, body composition, obesity

\section{INTRODUCTION}

Insulin resistance plays a significant role in the etiopathogenesis of sarcopenia, which is defined as age-related progressive loss of muscle mass and muscle strength (I-3). It is estimated that sarcopenic obesity will increasingly gain importance in the next decade due to the increasing prevalence of obesity, which is one of the most important risk factors of insulin resistance $(3,4)$. Although previous studies about sarcopenia have usually been conducted with elder people, thinking that the loss of muscle mass starts after the third decade, the diagnosis of sarcopenia might be underestimated in middle-aged people $(3,5)$. In addition, there are limitations regarding the evaluation of muscle mass in obese participants due to high body mass index (BMI) and fat percentage (6-8). Another difficulty in diagnosing sarcopenia is the use of many formulas for evaluation of muscle mass and, accordingly, the variety of the prevalence of sarcopenia (I, 5). While some studies use extremity muscle mass to evaluate sarcopenia, others use total muscle mass (5). Nevertheless, some formulas calculate the ratio of muscle mass to body weight, whereas other formulas calculate the ratio of muscle mass to height (5). The aim of the present study was to evaluate the prevalence of sarcopenia using different formulas in patients with prediabetes. 


\section{MATERIAL and METHODS}

The present study was conducted with data derived from the project entitled "Evaluation of muscle mass in obesity, prediabetes, and diabetes mellitus by different equations used for diagnosis of sarcopenia." Participants with prediabetes aged 18-65 years who visited the Kartal Dr Lütfi Kırdar Training and Research Hospital obesity outpatient clinic between 2013 and 2015 were retrospectively evaluated. In the present study, participants with fasting plasma glucose (FPG) of $100-125 \mathrm{mg} / \mathrm{dL}$, glycated hemoglobin of $5.7 \%-6.4 \%$, or 2-hour plasma glucose level of 140-199 mg/dL on a 75-gram oral glucose tolerance test were considered as prediabetics (9). The study was approved by the ethics committee of Kartal Dr Lütfi Kırdar Training and Research Hospital (approval no.: 89513307//009/537-II8).

\begin{tabular}{|c|c|c|c|}
\hline & Female $(n=33 \mid)$ & Male ( $n=193)$ & $p^{*}$ \\
\hline Age (year) & & & 0.416 \\
\hline $\mathrm{BMI}\left(\mathrm{kg} / \mathrm{m}^{2}\right)$ & $41.35 \pm 11.54$ & $40.47 \pm 12.27$ & 0.013 \\
\hline Total muscle mass $(\mathrm{kg})$ & $38.80 \pm 5.71$ & $37.49 \pm 5.82$ & $<0.00$ \\
\hline $\operatorname{ALM}(\mathrm{kg})$ & $29.44 \pm 4.20$ & $43.43 \pm 6.35$ & $<0.00$ \\
\hline $\mathrm{SMI}\left(\mathrm{kg} / \mathrm{m}^{2}\right)$ & $26.52 \pm 4.26$ & $35.55 \pm 6.34$ & $<0.00$ \\
\hline ALM/height ${ }^{2}\left(\mathrm{~kg} / \mathrm{m}^{2}\right)$ & $11.76 \pm 1.48$ & $|4.5| \pm \mid .82$ & $<0.00$ \\
\hline MMR (\%) & $10.61 \pm 1.68$ & $11.85 \pm 1.75$ & $<0.00$ \\
\hline ALM/BMI & $30.57 \pm 3.26$ & $39.15 \pm 4.92$ & $<0.00$ \\
\hline \multirow[t]{2}{*}{ Fat percentage (\%) } & $0.686 \pm 0.077$ & $0.953 \pm 0.133$ & $<0.00$ \\
\hline & $41.22 \pm 3.43$ & $33.28 \pm 5.43$ & \\
\hline \multicolumn{4}{|c|}{$\begin{array}{l}\text { ALM: appendicular lean mass; BMI: body mass index; MMR: muscle } \\
\text { mass ratio; SMl: skeletal muscle index } \\
\text { *Student t-test } \\
\text { All data are presented as mean } \pm \text { standard deviation }\end{array}$} \\
\hline
\end{tabular}

TABLE 2. The frequency of sarcopenia among genders according to the formulas used for diagnosis of sarcopenia

Female $(n=331) n(\%)$

Male $(n=193) n(\%)$

$\mathrm{ALM} /$ height $^{2}\left(\mathrm{~kg} / \mathrm{m}^{2}\right)$

$\begin{array}{lcc}\text { Normal } & 331(100) & 192(99.48) \\ \text { Sarcopenia } & 0(0.00) & \text { I (0.52) }\end{array}$

ALM/BMI

Normal

$331(100)$

$176(91.19)$

Sarcopenia

$0(0.00)$

$17(8.81)$

$\mathrm{SMI}\left(\mathrm{kg} / \mathrm{m}^{2}\right)$

Normal

$331(100)$

192 (99.48)

Mild sarcopenia

$0(0.00)$

I (0.52)

Severe sarcopenia

$0(0.00)$

$0(0.00)$

$\operatorname{MMR}(\%)$

Normal

27I (81.87)

130 (67.36)

Mild sarcopenia

59 (17.83)

52 (26.94)

Severe sarcopenia

I (0.30)

II (5.70)

ALM: appendicular lean mass; BMI: body mass index; MMR: muscle mass ratio; SMl: skeletal muscle index

Measurement methods: After a 12-hour fasting period, all participants were evaluated by a bioelectrical impedance analysis (BIA) device (GAIA 359 PLUS; Jawon Medical, Korea; 20II) with regard to height, body weight, the sum of appendicular lean masses (ALMs) of the four limbs, impedance, and fat percentage. Thereafter, BMI was calculated as body weight in kilograms divided by the square of the height in meters, and total body mass was calculated using the formula: [(height ${ }^{2}(\mathrm{~cm}) /$ $\mathrm{BIA}$ resistance $\times 0.40 \mathrm{I})+($ gender $\times 3.825)+($ agex-0.07I) $]+5.102$ (I0) . A skeletal muscle index (SMI), which is calculated as the total muscle mass divided by the square of the height in meters, of $\leq 5.75 \mathrm{~kg} / \mathrm{m}^{2}$ is considered as severe sarcopenia and $5.76-6.75$ $\mathrm{kg} / \mathrm{m}^{2}$ is considered as mild sarcopenia in female patients, whereas an SMI of $\leq 8.50 \mathrm{~kg} / \mathrm{m}^{2}$ is considered as severe sarcopenia and $8.51-10.75 \mathrm{~kg} / \mathrm{m}^{2}$ is considered as mild sarcopenia in male patients (II). Muscle mass ratio (MMR) is the percentage of total muscle mass divided by body weight, where $<22.1 \%$ is defined as severe sarcopenia and $22.1 \%-27.6 \%$ is defined as mild sarcopenia in female patients and $<31.5 \%$ is defined as severe sarcopenia and $31.5 \%-37.0 \%$ is defined as mild sarcopenia in male patients (12). With regard to the other formulas used for diagnosis of sarcopenia, the cut-off value for ALM/BMI ratio is considered to be 0.512 in female patients and 0.789 in male patients, and the cut-off value for ALM/height ${ }^{2}$ ratio is considered to be $5.45 \mathrm{~kg} / \mathrm{m}^{2}$ in female patients and $7.26 \mathrm{~kg} / \mathrm{m}^{2}$ in male patients $(13,14)$. Moreover, FPG of participants was measured by hexokinase method, whereas fasting insulin level was measured by chemiluminescent immunoassay; thereafter, homeostatic model assessment of insulin resistance (HOMA-IR) was calculated using the formula: FPG (mg/dL) xfasting insulin $(\mu \mathrm{U} /$ $\mathrm{mL}) / 405$ (15).

Exclusion criteria: Patients with type I diabetes mellitus, hyperthyroidism, chronic renal failure, chronic liver failure, and documented neuromuscular disease and pregnant women were excluded from the study. In addition, participants $>65$ years were also excluded as aging is a risk factor for sarcopenia.

Sample size: The sample size was calculated using the prevalence of $50 \%$, margin error of $5 \%$, confidence level of $95 \%$, and missing data of $20 \%$. The target sample size was determined as 461 patients.

\section{Statistical Analysis}

Data were performed by Statistical Package for the Social Sciences version 22.0 program (IBM Corp.; Armonk, NY, USA).Descriptive statistics were presented as frequency, percentage, mean \pm standard deviation, and median (minimum-maximum). Student's t-test was used for normally distributed continuous variables. Mann-Whitney $U$ test was used for comparison of continuous variables with non-normal distribution. Pearson's correlation test was used to assess the linear association between two variables. A p value of $<0.05$ was considered as significant for all results.

\section{RESULTS}

Of 524 participants with prediabetes, 33I (63.17\%) were female. The mean HOMA-IR levels were 4.67 (0.78-22.77) in female and $5.53(1.50-35.38)$ in male patients $(p<0.001)$. Distributions of age and bioimpedance measurements among genders are summarized in Table I. 
TABLE 3. Participants without and with sarcopenia with regard to age, HOMA-IR, and BMI values by using MMR



Sarcopenia was found in 60 (18.13\%) female patients using MMR, whereas no sarcopenia was detected using SMI. Sarcopenia was detected in 63 (32.64\%) male patients using MMR and in I (0.52\%) male patient using SMI. The frequency of sarcopenia among genders according to the formulas used for diagnosis of sarcopenia is summarized in Table 2.

There was no significant difference between participants without and with sarcopenia of each gender with regard to age, HOMA-IR, and BMI values when sarcopenia was assessed using SMI, ALM/BMI ratio, and ALM/height ${ }^{2}$ ( $p>0.05$ ). Participants without and with sarcopenia with regard to age, $\mathrm{HO}$ MA-IR, and BMI values by using MMR are summarized in Table 3. Additionally, a significant relationship was observed between age and MMR in both genders $(r=-0.148, p=0.040$ for female and $r=-0.156, p=0.004$ for male).

\section{DISCUSSION}

The present study aimed to evaluate the prevalence of sarcopenia in patients with prediabetes using different formulas. While the prevalence of sarcopenia in female patients was $18.13 \%$ using MMR, no sarcopenia was determined using SMI, ALM/BMI ratio, and $A L M /$ height $^{2}$. In male patients, the prevalence of sarcopenia was $32.64 \%$ using MMR, $0.52 \%$ using SMI, $8.81 \%$ using ALM/BMI ratio, and $0.52 \%$ using ALM/ height $^{2}$.

Previous studies about sarcopenia are usually being conducted in elder people, and various formulas are used to make the diagnosis (5). In a previous study, the prevalence of sarcopenia in female patients was determined as $23.6 \%$ using MMR and $2.8 \%$ using SMI (16). In another study, the prevalence of sarcopenia in 40-59-year-old female patients was 2.5\% using ALM/ height $^{2}$ and $4.2 \%$ using MMR (4). The prevalence of sarcopenia in elder women was found to be $18.9 \%$ using ALM/BMl ratio, which is one of the formulas established in recent years to assess muscle mass (I3). In the present study, MMR found mild sarcopenia in $17.83 \%$ and severe sarcopenia in $0.30 \%$ of female patients, whereas SMI, $\mathrm{ALM} / \mathrm{BMI}$, and ALM/height ${ }^{2}$ determined no sarcopenia. In previous studies evaluating the prevalence of sarcopenia in male patients, it was $0.0 \%-56.7 \%$ using $A L M /$ height $^{2}$ and $23.6 \%-68.0 \%$ using $\operatorname{SMI}(5,17)$. In a previous study, the prevalence of sarcopenia in 40-59-year-old male patients was found to be $2.8 \%$ using ALM/height ${ }^{2}$ and $1.4 \%$ using MMR (4). In another study, the prevalence of sarcopenia in male patients was determined to be $12.5 \%$ using MMR and $3.6 \%$ using SMI (16). Furthermore, the prevalence of sarcopenia in elder male patients was found to be $28.5 \%$ using ALM/BMI ratio, which is another formula (I3). In the present study, MMR revealed mild sarcopenia in $26.94 \%$ and severe sarcopenia in $5.70 \%$ of male participants. Nevertheless, the prevalence of sarcopenia was found to be $0.52 \%$ using SMI and $\mathrm{ALM} /$ height $^{2}$ and $8.81 \%$ using ALM/BMI ratio. In the present study, the reason for low prevalence of sarcopenia detected using SMI and ALM/height ${ }^{2}$ in both genders might be due to higher BMI level than in the literature. ALM/height ${ }^{2}$ shows a strong correlation with BMl; therefore, it does not appear to be an appropriate method for assessment of sarcopenia in obese participants (4). Total muscle mass or ALM after adjusted to BMI, fat mass, and height is recommended for evaluation of muscle mass in obese participants $(4,5)$. ALM/BMl ratio, which was developed for obese participants, determined lower prevalence of sarcopenia in each gender as compared with the literature. This might be due to young and middle-aged participants of the present study, which is different from other studies. The loss of muscle mass begins from nearly 40 years old and becomes apparent from 60 years old (5). In the present study, a higher prevalence of sarcopenia determined using MMR as compared with a population-based study conducted in patients with similar age might be due to the prediabetic study population of the present study, which is a risk factor for sarcopenia (4).

Although male patients have higher muscle mass, the loss of muscle mass with aging is also higher in male patients (2). While some studies performed in older people found the prevalence of sarcopenia to be higher in female patients using MMR, a previous study found it to be higher in male patients $(12,16,18)$. In addition, the prevalence of sarcopenia using SMI and ALM/BMI ratio was usually higher in male than in female patients $(5,11,13$, 16-18). In the present study, there was no difference between the genders with regard to the prevalence of sarcopenia using SMI and ALM/height; however, the prevalence of sarcopenia was found to be higher in male patients using MMR and ALM/BMI. This might have resulted from higher HOMA-IR in male patients, which is an indicator of insulin resistance $(I, 15)$.

Being one of the rare studies evaluating the prevalence of sarcopenia in patients with prediabetes is the strength of the present study. One of the limitations of the present study was the fact that the duration of prediabetes among the study participants was unknown, and the other limitation is the use of the BIA method to assess particularly ALM. Although dual energy $X$-ray absorptiometry (DEXA) or magnetic resonance imaging is recommended for measurement of muscle mass, $\mathrm{BIA}$ is preferred because it is a practical, portable and low-cost method (I0). There are formulas established to assess total muscle mass using BIA; however, previous studies usually prefer DEXA for measurement of $\operatorname{ALM}(5,10,13,17)$. 
In conclusion, the prevalence of sarcopenia in female participants with prediabetes was $18.13 \%$ using MMR, whereas no sarcopenia in female participants was found using SMI, ALM/BMI ratio, and ALM/height2. The prevalence of sarcopenia in male participants was $32.64 \%$ using MMR, $0.52 \%$ using SMI, $8.81 \%$ using ALM/BMI ratio, and $0.52 \%$ using ALM/height². The prevalence of sarcopenia shows wide variations even using ALM/ BMl ratio and MMR, which are recommended for assessment of muscle mass particularly in obese participants. As prediabetes and insulin resistance are significant risk factors for sarcopenia, new formulas are required to be developed for assessing muscle mass in young and middle-aged obese participants with prediabetes to eliminate the concealment of sarcopenia.

Ethics Committee Approval: Ethics committee approval was received for this study from the Ethics Committee of Kartal Dr Lütfi Kırdar Training and Research Hospital (Approval Date: 29.01.2016, Approval Number: 89513307/1009/537-||8)

Informed Consent: Informed consent is not necessary due to the retrospective nature of this study.

Peer-review: Externally peer-reviewed.

Author contributions: Concept - S.T., E.S., R.D.; Design - S.T., E.S.; Supervision - S.T., R.D.; Resource - S.T., E.O., I.T.; Materials - E.S., E.O., I.T.; Data Collection and/or Processing - S.T., E.S., E.O.; Analysis and/or Interpretation - S.T., E.S., I.T.; Literature Search - S.T., E.S., R.D.; Writing - S.T., E.S., R.D., E.O.; Critical Reviews - S.T., E.O., I.T., R.D.

Conflict of Interest: The authors have no conflicts of interest to declare.

Financial Disclosure: The authors declared that this study has received no financial support.

\section{REFERENCES}

I. Fielding RA, Vellas B, Evans WJ, Bhasin S, Morley JE, Newman AB, et al. Sarcopenia:an undiagnosed condition in older adults. Current consensus definition: prevalence, etiology, and consenquences. International working group on sarcopenia. J Am Med Dir Assoc 20II; 12: 249-56. [CrossRef]

2. Kim TN, Park MS, Yang SJ, Yoo HJ, Kang HJ, Song W, et al. Prevalence and determinant factors of sarcopenia in patients with type 2 diabetes: the Korean Sarcopenic Obesity Study (KSOS). Diabetes Care 2010; 33: 1497-9. [CrossRef]

3. Moon SS. Low skeletal muscle mass is associated with insulin resistance, diabetes, and metabolic syndrome in the Korean population: the Korea National Health and Nutrition Examination Survey (KNHANES) 2009-2010. Endocr J 2014; 61: 61-70. [CrossRef]
4. Kim TN, Yang SJ, Yoo HJ, Lim Kl, Kang HJ, Song W, et al. Prevalence of sarcopenia and sarcopenic obesity in Korean adults: the Korean sarcopenic obesity study. Int J Obes (Lond) 2009; 33: 885-92. [CrossRef]

5. Pagotto $\vee$, Silveira EA. Methods, diagnostic criteria, cutoff points, and prevalence of sarcopenia among older people. Scientific World Journal 2014; 2014: 231312. [CrossRef]

6. Thomas DR. Sarcopenia. Clin Geriatr Med 20I0; 26: 331-46. [CrossRef]

7. Park SW, Goodpaster BH, Lee JS, Kuller LH, Boudreau R, de Rekeneire $N$, et al. Excessive loss of skeletal muscle mass in older adults with type 2 diabetes. Diabetes Care 2009; 32: 1993-7. [CrossRef]

8. Lee SW, Youm Y, Lee WJ, Choi W, Chu SH, Park YR, et al. Appendicular Skeletal Muscle Mass and Insulin Resistance in an Elderly Korean Population: The Korean Social Life, Health and Aging Project-Health Examination Cohort. Diabetes Metab J 2015; 39: 37-45. [CrossRef]

9. American Diabetes Association. Classification and Diagnosis of Diabetes. Standards of Medical Care in Diabetes 20l6. Diabetes Care 2016; 39(Suppl I): 13-22.

10. Janssen I, Heymsfield SB, Baumgartner RN, Ross R. Estimation of skeletal muscle mass by bioelectrical impedance analysis. J Appl Physiol (1985) 2000; 89: 465-7I. [CrossRef]

II. Janssen I, Baumgartner RN, Ross R, Rosenberg IH, Roubenoff R. Skeletal muscle cutpoints associated with elevated physical disability risk in older men and women. Am J Epidemiol 2004; 159: 4I321. [CrossRef]

12. Janssen I, Heymsfield SB, Ross R. Low relative skeletal muscle mass (sarcopenia) in older persons is associated with functional impairment and physical disability. J Am Geriatr Soc 2002; 50: 889-96. [CrossRef]

13. Cheung CL, Lam KS, Cheung BM. Evaluation of Cutpoints for Low Lean Mass and Slow Gait Speed in Predicting Death in the National Health and Nutrition Examination Survey 1999-2004. J Gerontol A Biol Sci Med Sci 2016; 71: 90-5. [CrossRef]

14. Gallagher D, Visser M, De Meersman RE, Sepúlveda D, Baumgartner RN, Pierson RN, et al. Appendicular skeletal muscle mass: effects of age, gender, and ethnicity. J Appl Physiol (1985) 1997; 83: 229-39. [CrossRef]

15. Kozawa J, Inoue K, Iwamoto R, Kurashiki Y, Okauchi Y, Kashine S, ef al. Liraglutide is effective in type 2 diabetic patients with sustained endogenous insulin-secreting capacity. J Diabetes Investig 2012; 3: 294-7. [CrossRef]

16. Tichet J, Vol S, Goxe D, Salle A, Berrut G, Ritz P. Prevalence of sarcopenia in the French senior population. J Nutr Health Aging 2008; 12: 202-6. [CrossRef]

17. Chien MY, Huang TY, Wu YT. Prevalence of sarcopenia estimated using a bioelectrical impedance analysis prediction equation in community-dwelling elderly people in Taiwan. J Am Geriatr Soc 2008; 56: 1710-5. [CrossRef]

18. Castillo EM, Goodman-Gruen D, Kritz-Silverstein D, Morton DJ, Wingard DL, Barrett-Connor E. Sarcopenia in elderly men andwomen: the Rancho Bernardo study. Am J Prev Med 2003; 25: 22631. [CrossRef] 